\title{
Sex Differences in the Relationships Among Weight Gain, Subcutaneous Skinfold Tissue and Saltatory Length Growth Spurts in Infancy
}

\author{
MICHELLE LAMPL, AMANDA L. THOMPSON, AND EDWARD A. FRONGILLO \\ Department of Anthropology [M.L., A.L.T.], Emory University, Atlanta, GA 30322; Division of Nutritional \\ Sciences [E.A.F.], Cornell University, Ithaca, NY 14853
}

\begin{abstract}
ABST
As the mechanisms controlling the amount and timing of
growth saltations are not well understood, the identification of
physiologic coupling in weight and length growth are important
for further understanding normal growth biology. Thirty-four
healthy infants (13 males, 21 females) participated in a longitu-
dinal growth study during the first year. Weekly weights and s.c.
skinfolds (limb and trunk) were analyzed in a growth event-
focused study. Coincident analysis tested the null hypothesis of
chance concurrence between significant weight gain and salta-
tory length growth spurts. Logistic regression quantified this
relationship and investigated the interaction between incremental
weight gain and s.c. skinfolds on length growth spurts. The null
hypothesis of random coincidence between weight gain and
saltatory length growth was not supported. For girls, significant
\end{abstract}
While weight and height growth have often been considered to be physiologically coupled (1), how weight gain is related to linear growth during normal infant development is not well understood. As the proximal mechanisms controlling discrete growth spurts are unknown, identifying metabolic influences from weight changes may be important evidence for better understanding the normal biology of growth.

Variability in the sequence of weight and height growth patterns have been reported among nutritionally challenged samples. For example, studies of catch-up growth in previously malnourished children have observed that, with the onset of supplementation, repletion of weight and/or weight for height preceded linear growth (2-4). Seasonality studies have, likewise, identified alternating weight and height gains among infants and young children (5). These observations have been reiterated in an animal model that identified immediate weight

Received November 22, 2004; accepted May 4, 2005.

Correspondence: Michelle Lampl, M.D., Ph.D., Department of Anthropology, 1557 Dickey Drive, Emory University, Atlanta, GA 30322: e-mail: mlampl@emory.edu

Data were collected with support from the Developmental Psychobiology Research Group and the Wenner-Gren Foundation.

DOI: 10.1203/01.pdr.0000184327.65102.a6 weight gain and length growth were coupled during the same week and length saltations were $42 \%$ more likely during the weeks of significant weight gain, with no interaction from s.c. skinfolds. For boys, length growth saltations were coupled to both previous and concomitant weight gain but were predicted only by previous weight gain, controlling for confounders. Boys were $68 \%$ more likely to grow in length the week following significant weight gain, and initial abdominal to suprailiac skinfold ratios conferred a 4-fold increased likelihood of length growth within the week, controlling for confounders. These data generate the hypothesis that a common growth signal cascade couples growth in weight and length/height with a time delay due to sex-specific biology, reflected in a s.c. fat fold interface. (Pediatr Res 58: 1238-1242, 2005) gain in short-term fasted male rats after repletion, which preceded linear growth rate accelerations in the tibia (6).

A logical proposition from these studies is that weight gain is a signal for linear growth. This is in line with a historical "filling and stretching" notion of the relationship between weight gain and longitudinal growth (7). An offset in the timing of weight gain and linear growth may permit adipose tissue accrual to prepare the organism for growth via an adipocyte endocrine mechanism (6). This viewpoint may, however, be biased by the nutritionally compromised circumstances of the samples. In healthy growing children, there may be multiple paths for fueling linear growth. Upregulating dietary intake and/or drawing from stored energy resources (body fat) are also possibilities. This may result in no weight change as energy is shifted from stored resources to build new tissue. At this time, the exact nature of cross talk between energy status and linear growth is unknown.

The present study aimed to investigate the temporal relationships between weight gain and length increments in healthy growing infants. The length variable used was saltatory growth spurts of total body length as previously established (8). During this study, weight and multiple anthropometric measurements of the trunk and limbs were taken at weekly intervals 
across the first year. The novel aspect of the present investigation is that the time-intensive data collection protocol permitted event-focused study of growth as it occurred rather than as a continuous variable that might obscure the true relationship between weight and length growth increments.

The first question addressed in this investigation concerned the temporal relationship between significant weight gain and saltatory length growth and tested whether they were concurrent or sequential. Second, to investigate whether weight gain was merely a proxy for body energy stores, the interaction between incremental weight gain and s.c. skinfolds on length growth spurts was explored. As sex differences in weight and skinfold thickness have been documented from fetal life $(9,10)$, the analyses were stratified by sex.

\section{METHODS}

Subjects and measurement protocol. Thirty-four infants (13 males, 21 females; 32 white, one Hispanic, and one African American) participated in a longitudinal growth study for durations ranging from 4 to 12 mo after parental informed, written consent of a Human Subjects' Committee approved protocol. During home visits, measures of the unclothed infants' weight were assessed weekly before eating (or $2 \mathrm{~h}$ after the last intake) with a Homs balance scale to the nearest $0.5 \mathrm{oz}$, calibrated before each measurement for a total of 1156 weekly weight measurements. All measurements were converted to grams for data entry. Seven limb and trunk skinfolds were measured at these times by the same observer (M.L.) with Holtain skinfold calipers (triceps, quadriceps, calf, subscapular, suprailiac, mid-axial, and abdominal) according to standard techniques (11). A pilot study established the intraobserver technical error of measurement for all skinfolds as $<0.2 \mathrm{~mm}$ based on 52 infants each measured two times with a 3-s compression time. This protocol was identified as the tolerance limit for multiple skinfold measures in a serial study of infants with measurement errors in line with previously published studies (12). Semiweekly $(n=21)$ and weekly $(n=13)$ body length measurements were taken according to the maximal stretch technique as previously reported (13). Daily parental diaries included real-time records of dietary intake and illness episodes of the infants (respiratory, gastrointestinal, atopic events and pediatrically diagnosed ear infections). The signs and symptoms of illness were verified by the researcher during home visits.

Data analysis. Previous research has documented a number of effects that may confound infant weight gain, including birth weight, gestational age, sex, feeding style, and illness episodes (14-16). The significance of each of these confounders was first investigated in separate models employing a mixedmodel regression with weight velocity $z$ score as the continuous outcome variable, individual as the random effect, and each of the potential confounders as a fixed effect (xtreg, STATA 8). Feeding style and illness were coded as bivariate variables (presence/absence of predominant breast-feeding and presence/absence of illness during the measurement interval). As a continuous variable, all weight velocities were expressed as grams per day to account for the lack of exact 7-d intervals between measurements for all infants. All tests of significance were two tailed.

To test for significant co-occurrence between length saltations and weight increments, a trichotomous variable for weight change was derived. Significant weekly weight change was defined as $>75 \mathrm{~g}$ determined from a pilot study (unpublished data) as the $95 \%$ reliability for weight change unaffected by intradaily bodily processes (urination, defecation, variations due to eating, or other diurnal activities). Thus, significant increments or decrements were weekly changes $>75 \mathrm{~g}$.

Because the saltatory length growth spurts of these infants are known to have occurred within a time frame shorter than $1 \mathrm{wk}(8)$, the resolution of any temporal linkage between weight velocity and length growth depends on the timing between growth assessment and actual growth. Thus, a sequence of weight change and length growth might be evident either within a single measurement interval or with a time lag of one measurement interval with weight preceding length. Both possibilities were tested in separate analyses with the null hypothesis that correspondence between significant changes in weight and length were random. In theory, the relationship between weight and length could be either positive or negative, with length growth spurts requiring previous weight gain, accompanying weight gain, or leading to weight loss.

A coincident analysis approach based on specific probability algebra was used to test the null hypotheses that significant weight change preceded and/or was coincident with a length increment by chance (Hypergeo, Pulse-XP
Michael Johnson, University of Virginia) (17). Computer simulations calculated the expected means, variances, and probability distributions for purely random concordance between two independently regulated processes (weight gain and length increment). This method addresses problems associated with repeated-measures design and autocorrelation that can introduce spurious cross-correlational influences. The null hypothesis of random coincidence was rejected in the present study when it was 5\% likely that the number of coincidences were due to chance alone. If this condition was met, it was likely that some kind of temporal coupling between the two processes (weight and length growth) could be inferred. The frame-shift analyses were conducted excluding the occurrence of length growth spurts in the preceding week to rule out the confounding of concordance. Analyses were run separately for significant weight increments and decrements.

This analysis was followed by a mixed-model logistic regression testing for significant weight change effects on body length growth spurts during the week as a dichotomous outcome variable (growth/no growth), with the individual as a random effect and age, breast-feeding, presence of concurrent illness, and initial weight as fixed covariates (xtlogit, STATA 8). This approach was used to provide a quantitative estimate of the relationship between significant weight gain and length growth spurts, if present. The mixed-model approach permitted consideration of other unmeasured individual-level confounding effects such as maternal prenatal milieu, genetically based metabolic differences, or other unknown confounders that may have contributed to intersubject variability.

The question of whether weight itself was important or whether weight gain was a proxy for changes in body composition associated with "fueling up" and shifting energy resources to growth was addressed by entering skinfold measurements as covariates in the preceding model. This was explored for each skinfold as well as the abdominal-to-suprailiac skinfold ratio, a variable chosen based on the documented metabolic importance of these sites $(18,19)$. Age, breast-feeding, presence of concurrent illness, and initial skinfold measures were included as fixed covariates. All analyses were stratified by sex.

\section{RESULTS}

After confirming normality of the data, the mean weights and lengths (Table 1) were compared with the U.S. sex-specific growth reference (Centers for Disease Control and Prevention, 2000). The sample was unremarkable in length, weight, and weight for length measurements throughout the first year of life. Boys' weight for length followed the median throughout the first year, with a mean length corresponding to the 50th percentile from birth to 6 mo of age, rising to the 75th percentile through the end of the first year. Their weight was at the 40th percentile at birth, then tracked the median from 3 to 9 months. The mean weight for length among girls was at the reference median at birth, dropped to the 25th percentile from 3 to 6 months, and matched the median from 9 mo. The mean female weight followed the median until 7 mo of age, then dropped to the 35th percentile at 9 mo for the remainder of the year. The mean female length followed the median throughout the first year.

There were significant sex differences in weight at birth controlling for gestational age (males $>$ females, $p=0.04$ ). By

Table 1. Weight and length by sex and age

\begin{tabular}{cccccc}
\hline & \multicolumn{2}{c}{ Boys $(n=13)$} & & \multicolumn{2}{c}{ Girls $(n=21)$} \\
\cline { 2 - 3 } \cline { 5 - 6 } $\begin{array}{c}\text { Age } \\
(\text { mo })\end{array}$ & $\begin{array}{c}\text { Weight }(\mathrm{kg}) \\
(\text { mean } \pm \mathrm{SD})\end{array}$ & $\begin{array}{c}\text { Length }(\mathrm{cm}) \\
(\text { mean } \pm \mathrm{SD})\end{array}$ & & $\begin{array}{c}\text { Weight }(\mathrm{kg}) \\
(\text { mean } \pm \mathrm{SD})\end{array}$ & $\begin{array}{c}\text { Length }(\mathrm{cm}) \\
(\text { mean } \pm \mathrm{SD})\end{array}$ \\
\hline Birth & $3.45 \pm 0.35^{*}$ & $51.1 \pm 2.4$ & & $3.34 \pm 0.39$ & $49.3 \pm 4.8$ \\
3 & $6.06 \pm 0.92 \dagger$ & $61.3 \pm 2.0$ & & $5.43 \pm 0.64$ & $60.0 \pm 2.4$ \\
6 & $7.93 \pm 0.11 \dagger$ & $69.2 \pm 1.6 \dagger$ & & $6.89 \pm 0.87$ & $66.1 \pm 1.9$ \\
9 & $8.97 \pm 0.53 \dagger$ & $73.2 \pm 1.8 \dagger$ & & $7.91 \pm 0.98$ & $70.4 \pm 1.8$ \\
12 & $9.80 \pm 0.53 \dagger$ & $76.7 \pm 1.9$ & & $8.96 \pm 0.94$ & $75.1 \pm 2.1$ \\
\hline
\end{tabular}

* Denotes significant sex differences with males larger; $p \leq 0.05$.

$\dagger$ Denotes significant sex differences with males larger; $p \leq 0.01$. 
$10 \mathrm{~d}$, these sex differences were no longer evident, but they re-emerged at 2 mo of age and continued across the first year. Although there were no significant sex differences in length before $3 \mathrm{mo}$, males were longer than females from 6 to $9 \mathrm{mo}$, ending the second half of the first year of life both longer and heavier than females (Table 1). Breast-feeding was initiated by 24 of the 34 mothers; 16 continued to breast-feed for at least 4 mo.

Weight-gain patterns were episodic and nonlinear as previously described for infants during their first year when measured at frequent intervals (20). Controlling for age and weight at the beginning of a week, weekly weight velocity was greater among males than females $(p=0.000)$ and breast-feeding was associated with lower weight gains, controlling for sex, age, and weight at the previous week $(p=0.02)$, consistent with previous research (16).

Temporal relationship between weekly weight velocity and length growth spurts. For the sample, significant weekly weight increments were found to be coincident with saltatory length growth spurts during the same week with a probability of coincidence by chance alone of $2.0 \times 10^{-5}$ (Fig. 1). Stratified by sex, both males and females exhibited this pattern with the concordance between weight and length growth greater than expected by chance alone with probabilities of 0.01 and 0.0002 for males and females, respectively.

Significant relationships were also found between weight gain in the week before a length growth spurt for males by coincident analysis with a probability of 0.03 that this was due to chance alone. No such relationship was identified for females. Length growth was not associated more frequently than expected by chance alone with weight loss during either time frame for either sex.

Logistic regression also identified sex differences in the timing between weight gain and length growth spurts (Table 2). For females, saltatory length growth spurts were positively predicted by concomitant significant weight gain: Girls were $42 \%$ more likely to grow in length during the week they gained significantly in weight than in the weeks they did not. Males, by contrast, showed a trend in concomitant predictability with weight as the single predictor [odds ratio (OR) $=1.29, p=$ $0.10]$, but when confounders were added to the model, no coincident predictability was identified. Males were, however, $68 \%$ more likely to grow in the week following significant weight gain, controlling for confounders $[\mathrm{OR}=1.68,95 \%$ confidence interval $(\mathrm{CI})=1.04-2.7, p=0.03]$.

The effect of s.c. skinfold measurements on the relationships between weight and length growth. Sex differences in the relationship between s.c. skinfolds and weight and length saltations were observed. No significant relationships between any s.c. skinfold measures and length growth spurts were found for females when these variables were entered as covariates. Among males, however, there was a trend for suprailiac skinfolds to positively predict growth within the following week. During the week of a growth spurt, the suprailiac skinfold was $20 \%$ more likely to decline than during weeks of no linear growth, with no effect from concurrent weight change. Moreover, the single best predictor of a growth spurt among males was the abdominal to suprailiac ratio at the onset of a week,
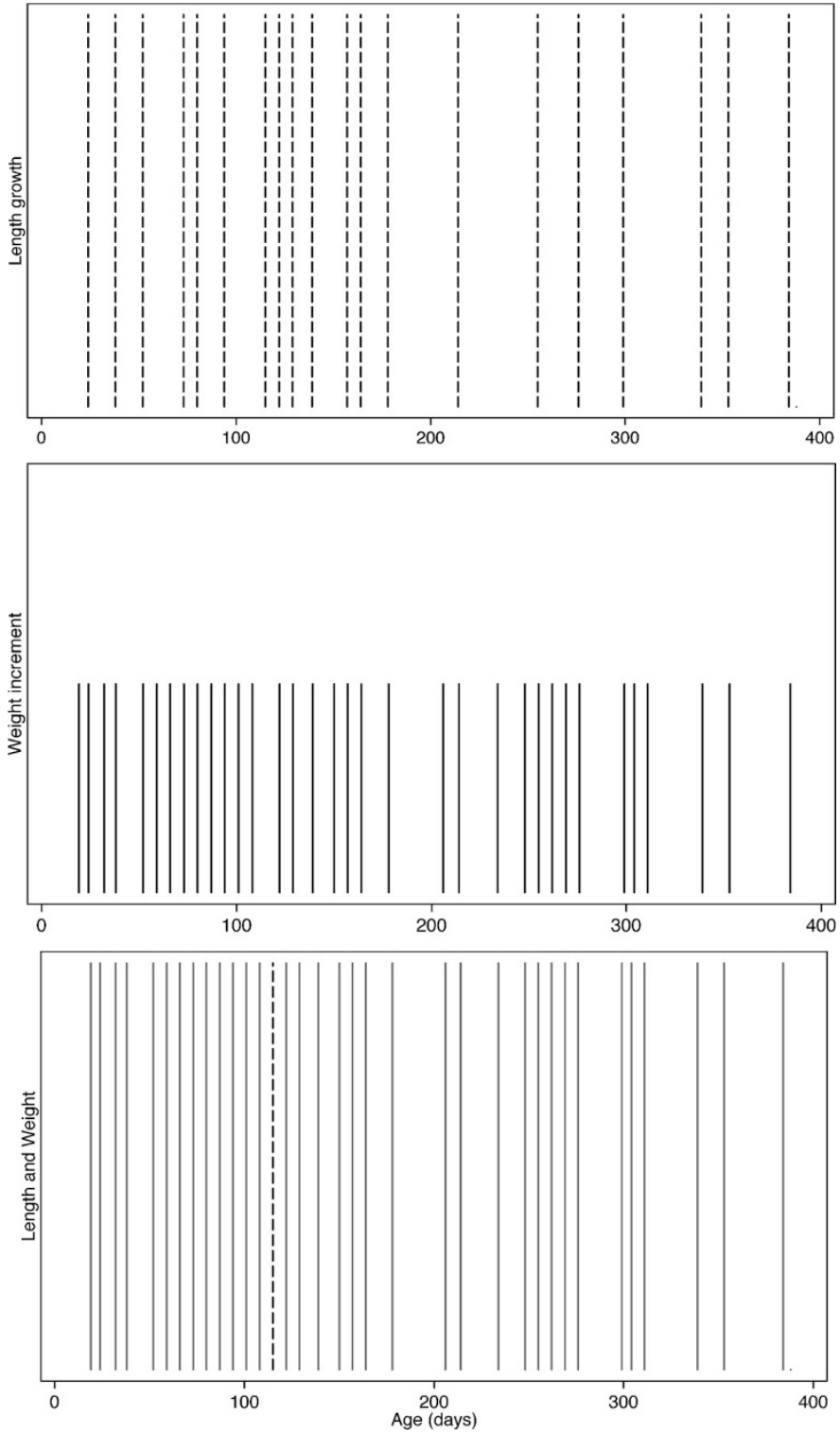

Figure 1. Saltatory length growth spurts (top) and significant weight gain (middle) are nonrandomly coincident (bottom) in this female infant over $56 \mathrm{wk}$ from the third week of life. During $34 \mathrm{wk}$, significant weight was gained and in $20 \mathrm{wk}$, significant length increments occurred; 19 of the length increments were coupled to significant weight gain. The probability that this was due to chance alone was 0.0003 .

which conferred a 4-fold greater odds of a length growth spurt in the coming week (Table 2).

In summary, sex modified the relationship between weight gain and length growth spurts in this sample. For girls, significant weight gain and saltatory length growth were coupled during the same week, with no predictive relationship between s.c. fat folds and linear growth. For boys, length growth spurts were coupled to both previous and concomitant weight gain, but were predicted only by previous weight gain, initial abdominal to suprailiac skinfold ratio and concomitant anthropometric shifts in suprailiac skinfold, controlling for confounders.

\section{DISCUSSION}

In this sample of healthy growing babies, nonrandom relationships between weight gain and saltatory length growth 
Table 2. Logistic mixed model results: predictors of saltatory length growth spurts

\begin{tabular}{|c|c|c|}
\hline $\begin{array}{l}\text { Main effect and } \\
\text { covariates }\end{array}$ & $\begin{array}{l}\text { Boys }(n=13) \\
(\mathrm{OR}, 95 \% \mathrm{CI})\end{array}$ & $\begin{array}{l}\text { Girls }(n=21) \\
(\mathrm{OR}, 95 \% \mathrm{CI})\end{array}$ \\
\hline $\begin{array}{l}\text { Week before growth spurt } \\
\text { Significant weight gain* }\end{array}$ & $1.68(1.04-2.7)$ & $0.95(0.67-1.3)$ \\
\hline \multicolumn{3}{|l|}{ Week of growth spurt } \\
\hline Significant weight gain $\dagger$ & $0.96(0.67-1.4)$ & $1.42(1.1-2.0)$ \\
\hline Significant weight gain $\dagger$ & $0.89(0.6-1.3)$ & $1.4(1.03-1.9)$ \\
\hline Suprailiac skinfold & $0.8(0.64-0.99)$ & $0.99(0.86-1.16)$ \\
\hline Suprailiac skinfold onset & $1.2(0.98-1.5)$ & $1.03(0.9-1.2)$ \\
\hline Significant weight gain $\dagger$ & $0.89(0.6-1.32)$ & $1.4(1.04-1.9)$ \\
\hline Suprailiac skinfold change & $0.22(0.05-0.91)$ & $0.8(0.3-2.2)$ \\
\hline Significant weight gain $\dagger$ & $0.84(0.56-1.25)$ & $1.4(1.02-1.9)$ \\
\hline Abdominal/suprailiac ratio & $4.3(1.1-17.2)$ & $0.68(0.28-1.7)$ \\
\hline
\end{tabular}

Significant models (variables $p<0.05$ ) are shown in bold.

* Model also controlled for age, concomitant illness, breastfeeding and weight at the beginning of the weekly interval.

$\dagger$ Model also controlled for age, concomitant illness, breast-feeding, weight, and skinfold thickness at the beginning of the weekly interval.

OR, odds ratio; CI, confidence interval.

spurts were identified, supporting a coupled process between weight and length growth. Sex differences in timing were identified, characterized by a s.c. skinfold interface among males and a more direct relationship among females.

Females' saltatory length growth spurts were both nonrandomly coupled to weight gain and significantly predicted from weight gain within a weekly interval after controlling for potential confounders, with no significant s.c. skinfold interactions. While a turnover in female skinfolds may have been more rapid than could be identified by the weekly measurements in this study, little evidence to date supports this alternative.

Among boys, coincident analysis identified both concomitant and lagged relationships between weight gain and length growth spurts. With the addition of confounders, however, logistic regression identified no significant predictive relationships between weight gain and saltatory length spurts within a week's measurement interval. Instead, weight gain predicted saltatory growth spurts in the following week. The reasons for the difference between the coincident analysis and logistic regression results for boys are unclear. This may identify a greater sensitivity to confounding effects among males, although a firm conclusion cannot be made with the modest sample size that was available.

These results suggest the possibility of distinction between males and females in aspects of the pathways leading to the growth signaling cascade in line with sex-based differences in hormones and body composition described for children (21). While there has been less attention paid to documenting growth hormone $(\mathrm{GH})$ pulsatility patterns in early infancy directly, normal growth rates from birth reflect intact $\mathrm{GH}$ pathways $(22,23)$, and sex differences in $\mathrm{GH}$ concentrations have been reported in neonates (24).

Sex-specific GH pulsatility patterns may coordinate body composition, weight gain, and linear growth. The higher growth rates characteristic of males have been associated with the more regular male-specific $\mathrm{GH}$ pulse patterns of low troughs and high peaks (25), while slowly growing children have been associated with the irregular female pulse pattern of high trough and low peak GH levels (26). A number of differential liver metabolic processes are driven by these sex differences in GH pulsatility (25) and may contribute to the growth rate differences through body composition and downstream hormonal effects (27).

The male pattern of weight gain, s.c. tissue, and length growth spurts found here is concordant with reports of an important role for abdominal adiposity in height growth among rapidly growing adolescent males (28). Previous studies have identified dimorphic responses of s.c. abdominal fat to growth hormone pulses (29-32). Taken together with endocrinological observations of leptin and GH pulse synchronicity, these observations further implicate a role for leptin in the growth cascade (33-36), if by paths influenced by sex steroids. Whether the loss of suprailiac s.c. skinfold attendant with length growth spurts among the infant boys was due to mechanical stretching, shifts in body composition, or the depletion of a high-turnover site for energy was not identifiable in this study.

The present results identified that weight gain was coupled to saltatory length growth spurts in both boys and girls and suggest that this may have involved a sex difference in timing associated with s.c. fat utilization. These observations may be relevant to previous conflicting reports regarding simultaneity versus. lag in the relationships between the timing of weight and height growth $(37,38)$, suggesting effect modification by sex differences and accompanying environmental sensitivities. Males' reliance on fat stores for growth has been reported to be greater than females in late gestation (10), and phase shifts in weight and length growth have been reported to characterize the male fetus by contrast with copulsatility, more common among females (39).

This study used data that were collected frequently to investigate relationships operating in very short time intervals. The number of children in the study was modest, but sufficient to have adequate power to identify meaningful differences and relationships and also to examine interactions of those relationships with sex through use of a stratified analysis. Because of the modest sample size of infants, the ability to examine other interactions with infant-specific factors was limited. These subjects were predominantly whiten and living under conditions that were not limiting to growth. Any extrapolation of these results to infants/children of other ethnic backgrounds and/or those living in poor environmental conditions should, therefore, be undertaken with caution.

Summary. The present data generate the hypothesis that a common growth signal cascade couples growth in weight and length/height. Furthermore, there may be a time delay due to sex-specific biology, reflected in a s.c. fat fold interface. Whether this is an additional step important in energy signaling or represents reliance on a shifting resources growth strategy requires further clarification. These observations may be a mechanistic basis for the observation that rapid infant growth carries with it subsequent health risk, particularly among males (40). This study suggests the need for further research into the metabolic aspects of discrete growth events to investigate whether the patterns observed among the infants in this sample 
occur more generally. Moreover, a focus on relationships among hormonal levels, body composition, and saltatory growth patterns may advance our understanding of normal growth biology and expand our appreciation of sex-specific effects.

\section{REFERENCES}

1. Waterlow JC 1994 Relationship of gain in height to gain in weight. Eur J Clin Nutr 48:S72-S73

2. Costello AM 1989 Growth velocity and stunting in rural Nepal. Arch Dis Child 64:1478-1482

3. Doherty CP, Sarkar MA, Shakur MS, Ling SC, Elton RA, Cutting WA 2001 Linear and knemometric growth in the early phase of rehabilitation from severe malnutrition. Br J Nutr 85:755-759

4. Walker SP, Golden MH 1988 Growth in length of children recovering from severe malnutrition. Eur J Clin Nutr 42:395-404

5. Maleta K, Virtanen SM, Espo M, Kulmala T, Ashorn P 2003 Seasonality of growth and the relationship between weight and height gain in children under three years of age in rural Malawi. Acta Paediatr 92:491-497

6. Farnum CE, Lee AO, O'Hara, K, Wilsman NJ 2003 Effect of short-term fasting on bone elongation rates: an analysis of catch-up growth in young male rats. Pediatr Res 53:33-41

7. Tanner JM 1981 A History of the Study of Human Growth. Cambridge University Press, Cambridge, p 499

8. Lampl M, Veldhuis JD, Johnson ML 1992 Saltation and stasis: a model of human growth. Science 258:801-803

9. Catalano PM, Drago NM, Amini SB 1995 Factors affecting fetal growth and body composition. Am J Obstet Gynecol 172:1459-1463

10. Guihard-Costa AM, Papiernik E, Grange G, Richard A 2002 Gender differences in neonatal subcutaneous fat store in late gestation in relation to maternal weight gain. Ann Hum Biol 29:26-36

11. Frisancho AR 1990 Anthropometric Standards for the Assessment of Growth and Nutritional Status. University of Michigan Press, Ann Arbor, p 189

12. Ulijaszek SJ, Kerr DA 1999 Anthropometric measurement error and the assessment of nutritional status. Br J Nutr 82:165-177

13. Lampl M, Birch L, Picciano MF, Johnson ML, Frongillo EA Jr 2001 Child factor in measurement dependability. Am J Hum Biol 13:548-557.

14. Nelson SE, Rogers RR, Ziegler EE, Fomon SJ 1989 Gain in weight and length during early infancy. Early Hum Dev 19:223-239

15. Preece MA, Freeman JV, Cole TJ 1996 Sex differences in weight in infancy. Published centile charts for weights have been updated. BMJ 313:1486

16. Dewey KG 1998 Growth characteristics of breast-fed compared to formula-fed infants. Biol Neonate 74:94-105

17. Veldhuis JD, Johnson ML, Seneta E 1991 Analysis of the copulsatility of anterior pituitary hormones. J Clin Endocrinol Metab 73:569-576

18. Gravholt CH, Schmitz O, Simonsen L, Bulow J, Christiansen JS, Moller N 1999 Effects of a physiological GH pulse on interstitial glycerol in abdominal and femoral adipose tissue. Am J Physiol 277:E848-E854

19. Huang TT, Johnson MS, Gower BA, Goran MI 2002 Effect of changes in fat distribution on the rates of change of insulin response in children. Obes Res 10:978-984

20. Giani U, Filosa A, Causa P 1996 A non-linear model of growth in the first year of life. Acta Paediatr 85:7-13

21. Garnett SP, Hogler W, Blades B, Baur LA, Peat J, Lee J, Cowell CT 2004 Relation between hormones and body composition, including bone, in prepubertal children. Am J Clin Nutr 80:966-972
22. Wit JM, Van Unen H 1992 Growth of infants with neonatal growth hormone deficiency. Arch Dis Child 67:920-924

23. Pena-Almazan S, Buchlis J, Miller S, Shine B, MacGillivray M 2001 Linear growth characteristics of congenitally GH-deficient infants from birth to one year of age. J Clin Endocrinol Metab 86:5691-5694

24. Geary MP, Pringle PJ, Rodeck CH, Kingdom JC, Hindmarsh PC 2003 Sexual dimorphism in the growth hormone and insulin-like growth factor axis at birth. J Clin Endocrinol Metab 88:3708-3714

25. Udy GB, Towers RP, Snell RG, Wilkins RJ, Park SH, Ram PA, Waxman DJ, Davey HW 1997 Requirement of STAT5b for sexual dimorphism of body growth rates and liver gene expression. Proc Natl Acad Sci U S A 94:7239-7244

26. Achermann JC, Brook CG, Robinson IC, Matthews DR, Hindmarsh PC 1999 Peak and trough growth hormone $(\mathrm{GH})$ concentrations influence growth and serum insulin like growth factor-1 (IGF-1) concentrations in short children. Clin Endocrinol (Oxf) 50:301-308

27. Hindmarsh PC, Fall CH, Pringle PJ, Osmond C, Brook CG 1997 Peak and trough growth hormone concentrations have different associations with the insulin-like growth factor axis, body composition, and metabolic parameters. J Clin Endocrinol Metab 82:2172-2176

28. Malina RM, Koziel S, Bielicki T 1999 Variation in subcutaneous adipose tissue distribution associated with age, sex, and maturation. Am J Human Biol 11:189-200

29. Samra JS, Clark ML, Humphreys SM, MacDonald IA, Bannister PA, Matthews DR, Frayn KN 1999 Suppression of the nocturnal rise in growth hormone reduces subsequent lipolysis in subcutaneous adipose tissue. Eur J Clin Invest 29:1045-1052

30. Roemmich JN, Clark PA, Berr SS, Mai V, Mantzoros CS, Flier JS, Weltman A, Rogol AD 1998 Gender differences in leptin levels during puberty are related to the subcutaneous fat depot and sex steroids. Am J Physiol 275:E543-E551

31. Veldhuis JD, Roemmich JN, Rogol AD 2000 Gender and sexual maturationdependent contrasts in the neuroregulation of growth hormone secretion in prepubertal and late adolescent males and females-a general clinical research center-based study. J Clin Endocrinol Metab 85:2385-2394

32. Weltman A, Weltman JY, Hartman ML, Abbott RD, Rogol AD, Evans WS, Veldhuis JD 1994 Relationship between age, percentage body fat, fitness, and 24-hour growth hormone release in healthy young adults: effects of gender. J Clin Endocrinol Metab 78:543-548

33. Koutkia P, Canavan B, Johnson ML, DePaoli A, Grinspoon S 2003 Characterization of leptin pulse dynamics and relationship to fat mass, growth hormone, cortisol, and insulin. Am J Physiol Endocrinol Metab 285:E372-E379

34. Fors H, Matsuoka H, Bosaeus I, Rosberg S, Wikland KA, Bjarnason R 1999 Serum leptin levels correlate with growth hormone secretion and body fat in children. J Clin Endocrinol Metab 84:3586-3590

35. Ertl T, Funke S, Sarkany I, Szabo I, Rascher W, Blum WF, Sulyok E 1999 Postnatal changes of leptin levels in full-term and preterm neonates: their relation to intrauterine growth, gender and testosterone. Biol Neonate 75:167-176

36. Nagy TR, Gower BA, Trowbridge CA, Dezenberg C, Shewchuk RM, Goran MI 1997 Effects of gender, ethnicity, body composition, and fat distribution on serum leptin concentrations in children. J Clin Endocrinol Metab 82:2148-2152

37. Maleta K, Virtanen S, Espo M, Kulmala T, Ashorn P 2003 Timing of growth faltering in rural Malawi. Arch Dis Child 88:574-578

38. Tillmann V, Thalange NK, Foster PJ, Gill MS, Price DA, Clayton PE 1998 The relationship between stature, growth, and short-term changes in height and weight in normal prepubertal children. Pediatr Res 44:882-886

39. Lampl M, Jeanty P 2003 Timing is everything: a reconsideration of fetal growth velocity patterns identifies the importance of individual and sex differences. Am J Hum Biol 15:667-680

40. Singhal A, Lucas A 2004 Early origins of cardiovascular disease: is there a unifying hypothesis? Lancet 363:1642-1645 